EPJ Web of Conferences 53, 04006 (2013)

DOI: $10.1051 /$ epjconf/20135304006

(C) Owned by the authors, published by EDP Sciences, 2013

\title{
Hybrid analysis for the Telescope Array
}

\author{
D. Ikeda ${ }^{1, a}$, T. Abu-Zayyad ${ }^{2}$, M. Allen², E. Barcikowski ${ }^{2}$, H. Sagawa ${ }^{1}$, \\ B.T. Stokes ${ }^{2}$ and G.B. Thomson ${ }^{2}$ for the Telescope Array Collaboration ${ }^{b}$
}

\author{
1 Institute for Cosmic Ray Research, University of Tokyo, Kashiwa, Chiba, Japan \\ 2 University of Utah, Salt Lake City, Utah, US
}

\begin{abstract}
The Telescope Array (TA) experiment is the largest Ultra-High Energy Cosmic Ray (UHECR) hybrid detector which consists of three stations of Fluorescence Detectors (FDs) and 507 Surface Detectors (SDs). The coincidence events which observed both by FD and SD is referred as hybrid event. The geometry and energy of each extensive air shower observed by hybrid mode are reconstructed with much more accurate resolution than monocular reconstruction alone. The hybrid event candidates were searched for by comparison of the trigger times between FD and SD in the good weather days from May 2008 to September 2010. By this search, we found 1306 hybrid events for BR, 1051 events for LR and 905 events for MD. In this paper, the performance of the hybrid technique and the energy spectra measured by using hybrid events are presented.
\end{abstract}

\section{INTRODUCTION}

The Telescope Array (TA) experiment, located in the West Desert of Utah, is the largest ultra-high energy cosmic ray (UHECR) observatory in the Northern Hemisphere. The experiment operates three fluorescence detectors (FDs) in hybrid mode with an array of 507 scintillation surface detectors (SDs). The SD array is deployed on a square grid of $1.2 \mathrm{~km}$ spacing and covers a total area of about $700 \mathrm{~km}^{2}$ [1]. Three FD stations are located on the periphery of the SD array at Middle Drum (MD), Black Rock Mesa (MR) and Long Ridge (LR) [2]. The MD station consists of the 14 refurbished HiRes-I telescopes. The BR and LR stations each comprises 12 newly constructed telescopes that were specifically designed for TA. The hybrid operation was started from May 2008.

The coincidence events between those FD events and the SD array are referred as hybrid events. The hybrid data set is particularly suitable for the measurement of the UHECR energy spectrum. The geometry and energy of each extensive air shower (EAS) observed this way are constructed with excellent resolution, using the longitudinal calorimetric measurements from the FD and the additional timing information of the SD. These give much more accurate results than monocular reconstruction alone. Moreover, the exposure is obtained with great precision from the flat (in energy dependence), geometrically determined aperture of the SD array. In this paper, we present the developed hybrid technique both for newly constructed FDs (BR and LR) and transferred FD from HiRes (MD). The energy spectra measured by using this method is also presented.

\footnotetext{
ae-mail: ikeda@icrr.u-tokyo.ac.jp

${ }^{\mathrm{b}}$ For the full authorlist see Appendix "Collaborations" in this volume.
}

This is an Open Access article distributed under the terms of the Creative Commons Attribution License 2.0, which permits unrestricted use, distribution, and reproduction in any medium, provided the original work is properly cited. 


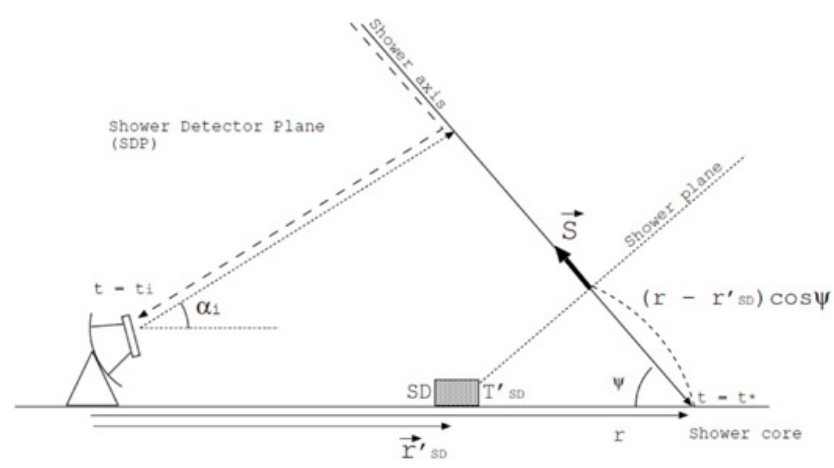

Figure 1. Shower detector plane and the time fit.

\section{SHOWER ANALYSIS}

\subsection{Analysis of BR and LR data}

The BR and LR FD sites are constructed specifically for the TA experiment. Each station has twelve telescopes: six telescopes are in the upper side, and the other six telescopes are in the lower side. The upper and lower rings cover $3^{\circ}-33^{\circ}$ in elevation and $108^{\circ}$ in azimuth. This field of view (FOV) covers the whole area of the SD array. Each telescope has a camera with 256 pixel photo-tubes and a spherical mirror with a diameter of $3.3 \mathrm{~m}$ which consists of 18 segment mirrors. Output signals from PMTs which pass through a DC-coupled pre-amplifier are continuously digitized by FADC on SDF with 12 bit and $40 \mathrm{MHz}$ sampling. Since each of 4 consecutive bins is combined to 1 bin in this module, it works as 14 bits and $10 \mathrm{MHz}$ sampling digitizer (100 ns/bin).

The hybrid event reconstruction for BR and LR sites is divided into two steps. First, the trajectory of the shower axis is determined from the pointing direction of FD pixels containing light pulse from the EAS, and from the combined FD and SD timing information. Second, the longitudinal development and total calorimetric energy of the shower are determined amount of light gathered by the FD.

The geometrical reconstruction is also divided into two parts. The shower-detector plane (SDP), which contains both the line of the shower axis and the point of the FD station, appears as a track in the PMT pixels of the camera (Fig. 1). The normal unit vector $\mathbf{n}$ of SDP can be found by searching for a least-squares solution, which minimizes $\sum_{i}\left(\mathbf{n} \cdot \mathbf{k}_{\mathbf{i}}\right)^{2}$, where $\mathbf{k}_{i}$ is the direction unit vector of the $i$-th PMT.

Next, the shower axis in the SDP is determined by timing and direction of the triggered PMTs, combined with the arrival time recorded by an SD counter near the SDP. The following equations are used to fit for $r$, the distance of the shower core on the ground from the FD station, where $\psi$ is the elevation angle of the shower axis in the SDP.

$$
\begin{gathered}
t_{i}=t^{*}+\frac{1}{c} \frac{\sin \psi-\sin \alpha_{i}}{\sin \left(\psi+\alpha_{i}\right)} r, \\
t^{*}=T_{S D}^{\prime}+\frac{1}{c}\left(r-r_{S D}\right) \cos \psi, \\
T_{S D}^{\prime}=T_{S D}-\frac{1}{c}\left\{\left(\mathbf{r}_{S D}^{\prime}-\mathbf{r}_{S D}\right) \cdot \mathbf{S}\right\} .
\end{gathered}
$$

Here $t_{i}$ and $\alpha_{i}$ are the time and the elevation angle in the SDP for the $i$-th PMT, $t^{*}$ is the time at which the air shower reaches the ground, $\mathbf{r}_{S D}$ is the position vector of the SD from the FD station, $\mathbf{r}_{S D}^{\prime}$ is the 


\section{UHECR 2012}
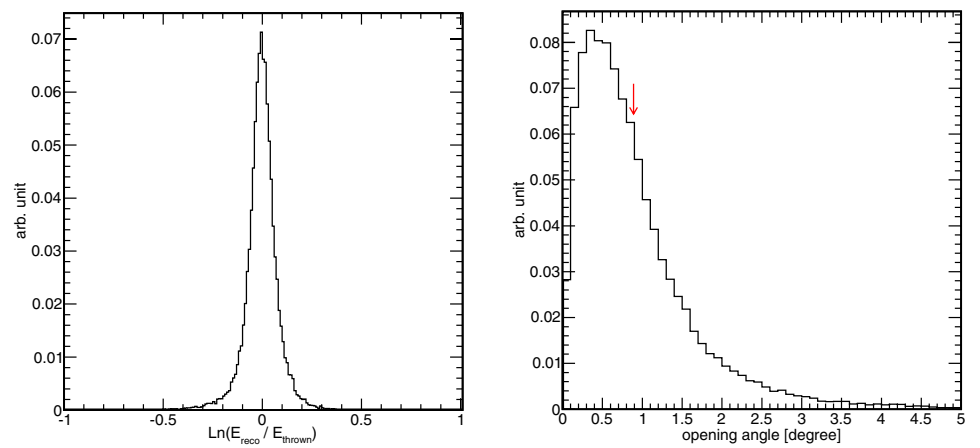

Figure 2. The energy resolution (left) and angular resolution (right) by the hybrid technique. The horizontal axis of the energy resolution figure is natural logarithm of the reconstructed energy over true simulation energy. That of the angular resolution figure is the opening angle between reconstructed direction and simulation direction. The vertical axes are the number of events.

SD position projected onto the SDP, and $\mathbf{S}$ is the direction vector of the shower axis at the shower core. Note that the time of the SD counter, $T_{S D}$, is included in the fit to improve the precision.

From the shower trajectory determined above, the pulse information from the time bins of PMTs involved in the event are projected on to the shower axis. From this we obtain a signal vs. shower depth curve. The shower profile (number of charged particles vs. shower slant depth) is obtained by an Inverse Monte Carlo (IMC) method. The total energy deposit along the shower axis is determined by the comparison between data and MC events generated by the Gaisser-Hillas (GH) function.

The calibration parameters are applied to MC in the IMC procedure. In this analysis, the differential fluorescence yield spectrum used to convert number of photons to energy deposit is taken from the published result of the FLASH experiment [3], normalized to the total yield from the Kakimoto model [4].

The calorimetric energy determined from the IMC procedure is corrected for missing energy carried off by neutral particles that do not emit fluorescence light. This "missing" energy is calculated using the air shower Monte Carlo (MC) simulations code CORSIKA [5]. This difference between primary energy and energy measured by the integration of the fitted GH function, is found to be $\sim 8 \%$. The energy of the primary particle is determined by integration of the fitted GH function with the correction of missing energy.

To ensure the quality of the reconstruction, we accept only events which satisfy the following quality criteria; (1) the number of PMTs used in reconstruction is more than 20, (2) the reconstructed zenith angle is smaller than 55 degrees, (3) shower core is inside the edge of SD array, (4) angle between reconstructed shower axis and telescope is more than 20 degrees, and (5) the maximum of the shower development $\left(\mathrm{X}_{\max }\right)$ has to be observed.

In the case of stereo event, if the events from both of BR and LR are accepted, the event with more PMTs is selected. For all energy ranges, the resolution of the arrival direction is about 0.9 degrees, and the resolution of the energy is on the order of $7 \%$ (see Fig. 2).

\subsection{Analysis of MD data}

The MD FD Site is instrumented with refurbished telescopes from the HiRes-1 site. Each telescope consists of $2 \mathrm{~m}$ diameter mirror of $3.7 \mathrm{~m}^{2}$ unobstructed light collection area. Each camera uses 256 twoinch photo-multiplier tubes (PMT) arranged in a row-wise hexagonal close-pack configuration. Each PMT pixel views a one-degree cone in the sky, and each camera has a $16^{\circ}$ (azimuth) $\times 14^{\circ}$ (elevation) field-of-view (FOV). Unlike the BR and LR sites, the read-out electronics are based on TDCs for 

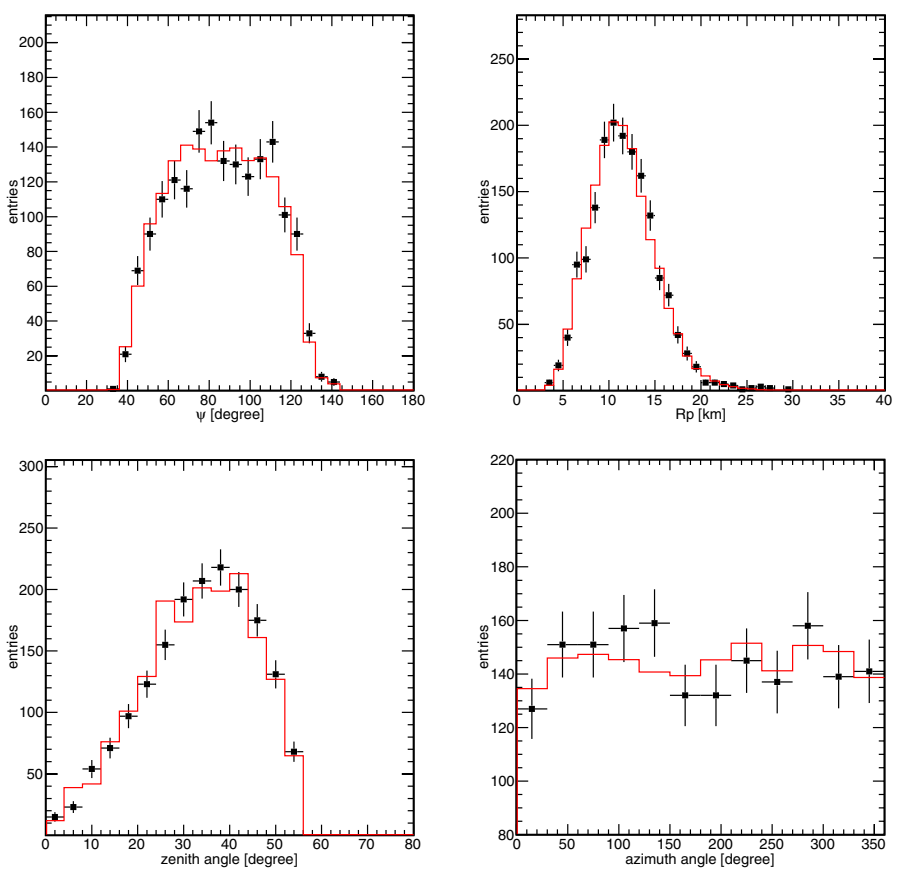

Figure 3. Comparison of the data and MC distributions in BR and LR events, $\psi$ (left upper), $R_{p}$ (right upper), zenith angle (left lower) and azimuth angle (right lower). The description of these parameters are in Figure 1.

time measurements of the light pulse, and on sample-and-hold charge integrators for pulse area determination.

For direct comparison to the HiRes results, the same profile-constrained fit from the Hires-1 monocular reconstruction was used for MD analysis. Details can be found in reference [6]. There are two main differences between the MD and HiRes-1 detectors. First, the MD station views up to $31^{\circ}$ in elevation, twice as high as HiRes-1, and covers only about $110^{\circ}$ in azimuth (full azimuth for HiRes). Second, the MD site is about 20\% darker in ambient background light, which permitted the detectors to run at a lower threshold, improving the aperture of the MD detector to about 1/2 that of HiRes-1, even though MD has less than $1 / 3$ the azimuthal coverage.

The FD reconstruction follows closely the technique used for the MD monocular spectrum [7], but without the profile constrain developed for the original HiRes-1 analysis. The addition of the timing from the SD incorporates the method described earlier in this paper. This study will be presented in another paper [8].

\subsection{Comparison of data and MC}

The hybrid event candidates were searched for by comparison of the trigger times between FD and SD in the good weather days from May 2008 to September 2010. By this search, we found 1306 hybrid events for BR, 1051 events for LR and 905 events for MD.

The MC data is also generated by using a CORSIKA based MC simulation code developed for TA [9]. For the detector response simulation for these MC events, all of the time-dependent calibration parameters were applied according to the relative exposure for the applicable periods. The MC event data were subjected to the same IMC reconstruction procedure and event selection cuts. For the purpose of validating our $\mathrm{MC}$ events, the reconstructed shower geometry is compared between observed data 

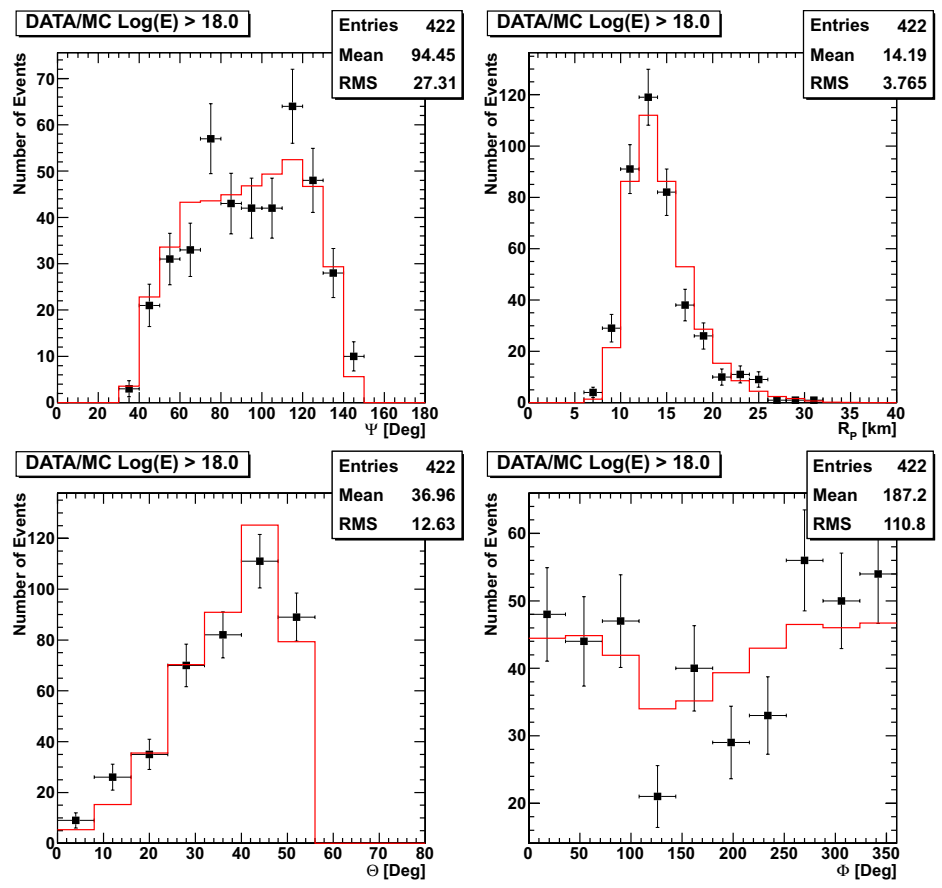

Figure 4. Comparison of the data and MC distributions in MD events. Each figure is corresponding to the plots in Figure 3.

and MC events (see Figs. 3 and 4). The MC events are in good agreement with the observed data. The effective exposure was calculated by using this MC data.

\subsection{Systematic uncertainty of energy determination}

The systematic uncertainties in energy determination are dominated by the uncertainties in the fluorescence yield (11\%), atmosphere attenuation (11\%) [10], the absolute detector calibration (10\%) [11-13], and the reconstruction procedure itself (10\%). The total systematic errors are estimated at $21 \%$ for the energy scale.

\section{ENERGY SPECTRUM}

The preliminary energy spectra from the hybrid events (BR and LR) in TA is shown in Figure 5. The updated MD monocular spectrum is also shown. These results from TA are consistent with the HiRes result [14]. This indicates that the energy scale of TA is compatible with that of HiRes.

\section{CONCLUSION}

The hybrid spectrum using the FD data from BR, LR and MD was presented. A dedicated reconstruction procedure was developed that included the timing information from the SD in the trajectory fit for the shower axis. The resulting angular resolution is better than $0.9^{\circ}$ and the energy resolution is about than $7 \%$ above $10^{18.2} \mathrm{eV}$. These resolutions represent a substantial improvement over FD monocular analysis, where the angular resolution is of the order of $5^{\circ}$ and energy resolution about $15-20 \%$ for events with the energy above $10^{18.2} \mathrm{eV}$. 


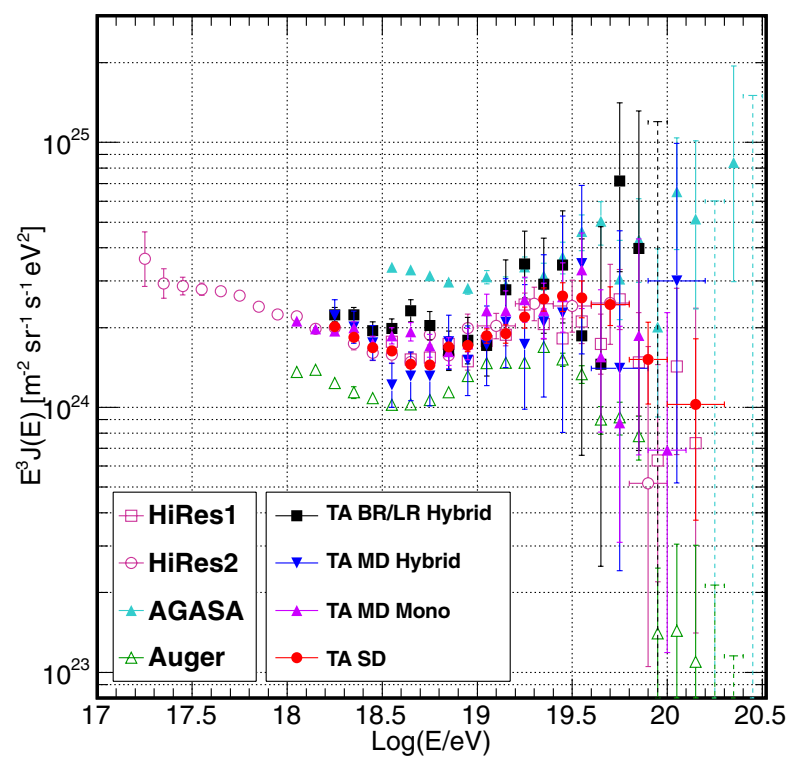

Figure 5. The energy spectra measured by the TA hybrid analysis with other recent experimental data. The dotted line shows the upper limit at $90 \%$ C.L.

For this hybrid analysis, the main contributions for the systematic uncertainty of energy scale are the detector calibration (10\%), atmospheric attenuation $(11 \%)$, fluorescence yield $(11 \%)$ and the reconstruction procedure itself $(10 \%)$. The total systematic error of the energy scale is estimated to be $21 \%$ by a quadratic sum of these factors.

The measured hybrid energy spectrum above $10^{18.2} \mathrm{eV}$ is consistent with the monocular spectrum from the MD site, and with the previously published results of the HiRes experiment.

The Telescope Array experiment is supported by the Japan Society for the Promotion of Science through Grantsin-Aid for Scientific Research on Specially Promoted Research (21000002) "Extreme Phenomena in the Universe Explored by Highest Energy Cosmic Rays", and the Inter-University Research Program of the Institute for Cosmic Ray Research; by the U.S. National Science Foundation awards PHY-0307098, PHY-0601915, PHY-0703893, PHY-0758342, and PHY-0848320 (Utah) and PHY-0649681 (Rutgers); by the National Research Foundation of Korea (2006-0050031, 2007-0056005, 2007-0093860, 2010-0011378, 2010-0028071, R32-10130); by the Russian Academy of Sciences, RFBR grants 10-02-01406a and 11-02-01528a (INR), IISN project No. 4.4509 .10 and Belgian Science Policy under IUAP VI/11 (ULB). The foundations of Dr. Ezekiel R. and Edna Wattis Dumke, Willard L. Eccles and the George S. and Dolores Dore Eccles all helped with generous donations. The State of Utah supported the project through its Economic Development Board, and the University of Utah through the Office of the Vice President for Research. The experimental site became available through the cooperation of the Utah School and Institutional Trust Lands Administration (SITLA), U.S. Bureau of Land Management and the U.S. Air Force. We also wish to thank the people and the officials of Millard County, Utah, for their steadfast and warm support. We gratefully acknowledge the contributions from the technical staffs of our home institutions and the University of Utah Center for High Performance Computing (CHPC).

\section{References}

[1] T. Abu-Zayyad et al., arXiv:1201.4964

[2] H. Tokuno et al., Nucl. Instr. and Meth. A 676, 54-65 (2012)

[3] R.U. Abbasi et al., AstroPart. Phys. 29, 77-86 (2007)

[4] F. Kakimoto et al., Nucl. Instr. and Meth. A 372, 527-533 (1996) 


\section{UHECR 2012}

[5] D. Heck et al., Report FZKA 6019 (1998)

[6] R.U. Abbasi et al., AstroPart. Phys. 23157 (2005)

[7] D. Rodriguez et al., arXiv:1202.5141v1

[8] M. Allen et al., Proc. 32 $2^{\text {nd }}$ International Cosmic Ray Conference 1313 (2011)

[9] B.T. Stokes et al., Astropart. Phys. 35, 759-766 (2012)

[10] T. Tomida et al., Nucl. Instr. and Meth. A 654, 653-660 (2011)

[11] H. Tokuno et al., Nucl. Instr. and Meth. A 601, 364-371 (2009)

[12] D. Ikeda et al., Proc. $31^{\text {st }}$ International Cosmic Ray Conference 0858 (2009)

[13] S. Kawana et al., Nucl. Instr. and Meth. A 681, 68-77 (2012)

[14] R.U. Abbasi et al., Phys. Rev. Lett. 100, 1011011 (2008) 